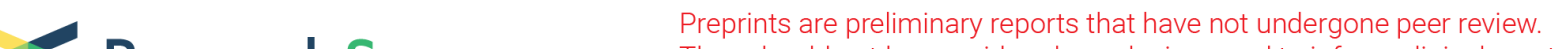 $\begin{array}{ll}\text { Research Square } & \text { They should not be considered conclusive, used to inform clinical practice, } \\ \text { or referenced by the media as validated information. }\end{array}$
}

\section{The Clinical Efficacy, Safety and Biomechanical Mechanism of Tai Chi in Patients with Knee Osteoarthritis: Study Protocol for a Randomized Controlled Trial}

\section{PAN FUWEI}

Shi's Center of Orthopedics and Traumatology, Shuguang Hospital Affiliated to Shanghai University of TCM https://orcid.org/0000-0002-0160-1495

\section{Min Zhang}

Institute of Traumatology \& Orthopedics, Shanghai Academy of TCM

\section{Guoqing Du}

Scientific Research Institute of Traumatology and Orthopedics: RSE Scientific Research Institute of Traumatology and Orthopedics

\section{Yuanchuan Chen}

Scientific Research Institute of Traumatology and Orthopedics

\section{Guangyue Yang}

Scientific Research Institute of Traumatology and Orthopedics: RSE Scientific Research Institute of Traumatology and Orthopedics

\section{Zhengyan Li}

Shi's Center of Orthopedics and Traumatology, Shuguang Hospital Affiliated to Shanghai University of TCM

Jiehang Lu

Scientific Research Institute of Traumatology and Orthopedics

Hongsheng Zhan ( $D$ pfw201208@163.com )

Shi's Center of Orthopedics and Traumatology, Shuguang Hospital Affiliated to Shanghai University of TCM https://orcid.org/0000-0002-3119-8596

\section{Study protocol}

Keywords: Knee osteoarthritis, Tai Chi, Effectiveness, Safety, Biomechanics.

Posted Date: March 15th, 2021

DOl: https://doi.org/10.21203/rs.3.rs-280199/v1 
License: (c) (i) This work is licensed under a Creative Commons Attribution 4.0 International License. Read Full License 


\section{Abstract}

Background: Knee osteoarthritis (KOA), one of the most common degenerative joint disease, is the leading cause of disability among elder people. Many previous studies demonstrated that Tai chi is an effective treatment in the management of KOA, but there has been a lack of study evaluated the effect of Tai chi on both clinical and biomechanical outcomes in individuals with KOA. The purpose of the study is to identify the clinical and biomechanical effect of the Tai chi, the results will help to improve the understanding of possible underlying biomechanical effects of the Tai chi.

Methods: We will conduct a prospective, randomized controlled trials of 72 individuals with medial KOA. The participants with medial KOA will be randomly divided into Tai chi group or a control group. The Tai chi group will participant in Tai chi twice a week for eight weeks. The control group will receive health care education. After the 8-week intervention period there will be a 16-week follow-period with no active intervention in either group. The primary and secondary outcomes will be assessed at baseline, 4 weeks, 8 weeks, 12 weeks and 24 weeks. The primary measure will be Western Ontario and McMaster Universities Osteoarthritis Index (WOMAC). The secondary outcome measures will include the kinetics, kinematics changing of the knee joint at sagittal, frontal, and transverse plane, the muscle co-contraction.

Discussion: This study aims to investigate the clinical and biomechanical effect of the Tai chi, and the results will help to improve the understanding of possible underlying biomechanical effects of the Tai chi in the management of KOA.

Trail Registration: The study was approved by China registered clinical trial ethics review committee with No.ChiECRCT20200335. The study plan was registered on the website of China Registered Clinical Trial Registration Center with No.ChiCTR2000040769 .Registered 09 December 2020, http://www.chictr.org.cn/showprojen.aspx?proj=65571

\section{Background}

\section{Background and rationale $\{6 a, 6 b\}$}

The main clinical symptoms of knee osteoarthritis (KOA) are joint stiffness, pain, poor joint activity, local tenderness, even joint deformity, loss of function and so on[1,2]. Recent studies have shown that KOA is not limited to cartilage and joint lesions, but a systemic psychosomatic disease caused by a variety of factors. First of all, KOA affects the whole body in the form of "Comorbidity". A study showed that patients with KOA had a higher risk of stroke than healthy volunteers and confirmed that this had nothing to do with the use of non-steroidal anti-inflammatory drugs [3]. An Australian study on KOA concluded that more than 50 per cent of KOA patients had hypertension, 20 per cent had cardiovascular disease, 19 per cent had dyslipidemia, 14 per cent had diabetes and 12 per cent had mental disorders such as depression [4]. Results of a systematic analysis show that one or more of these comorbidities or chronic diseases such as diabetes and cardiovascular disease also indicate increased pain or deterioration in KOA patients[5]. A cohort study also found that women with KOA symptoms had an increased risk of 
cardiovascular disease and mortality compared with women without KOA symptoms or signs [6]. Studies have shown that compared with people with healthy knee joints, KOA patients are more likely to develop depressive symptoms[7] and have stronger suicidal ideation [8]. Another study found a strong association between partial sleep, mood disorders, memory loss, and KOA[9]. Secondly, pain, as the first symptom and main treatment factor of KOA patients, seriously affects the physical and mental health of patients[10]. It has been found that KOA pain originates not only from peripheral nerve responses caused by joint and surrounding tissue injury or inflammatory stimulation of peripheral receptors [11, 12], but also from pain sensitization of central neurons caused by increased activity of descending fiber pain pathways or loss of anti-inflammatory effects in the central nervous system $[13,14]$. There has also been evidence in recent years that $[15,16]$, the source of KOA pain is not limited to peripheral and central nervous system sensitization, but also affected by a range of sociocultural and psychological factors, including underlying anxiety and depression [17].

To sum up, KOA is a systemic psychosomatic syndrome characterized by joint structural changes (such as cartilage loss, osteophyte formation), pain, joint movement disorders and other clinical symptoms, as well as psychological and social symptoms (such as anxiety, depression and other mental disorders). Chronic pain, physical disability and psychosocial diseases caused by KOA are one of the four major disability diseases in the world.As a non-fatal disease, if it is not treated in time and effectively, it will greatly reduce the quality of life of patients, and even lose their ability to work, which will bring serious mental and economic burden to their families and society. At present, almost all interventions for knee osteoarthritis are performed locally, including core therapies such as physiotherapy and muscle strengthening as recommended in the guidelines. However, the long-term curative effect is not satisfactory, and it is of little significance in improving patients' anxiety, pessimism, social efficiency and other psychosocial aspects.

Tai Chi is a multi-component intervention that integrates physical, psychological, emotional, mental and behavioral elements, which can have an impact on the body and spirit of patients, resulting in beneficial health outcomes[18]. Research has shown that Tai Chi exercises can improve muscle strength, proprioceptive sensitivity and neuromuscular activity, as well as physical and mental integration to reduce pain, thereby improving physical, mental and psychosocial health and overall quality of life, ideal for symptomatic KOA [19]. There is increasing evidence that Tai Chi exercise is beneficial to improve the clinical symptoms of patients with KOA, but there is still a lack of randomized controlled studies showing the clinical efficacy and safety of Taijiquan intervention in KOA patients, as well as the intervention of Tai Chi on lower limb biomechanics and gait motor function in KOA patients. Studies have shown that compared with normal people, patients with KOA not only show a high co-contraction ratio of flexion and extensor muscles during walking, but also have the phenomenon of over-activation of a single muscle. The contraction time is longer and the proportion of maximum spontaneous contraction is significantly increased in a complete gait cycle of the patients with KOA[20]. In addition, the weakening of the quadriceps femoris muscle, which plays a key role in maintaining the dynamic stability of knee joint, is also directly related to the abnormal synergistic contraction of flexion and extensor muscles of knee 
joint[21]. Lewek and some other people alike demonstrated a positive correlation between knee flexorextensor Muscle co-contraction ratio and clinical symptoms in KOA patients[22].

\section{Objective $\{7\}$}

Therefore, whether Tai Chi can regulate and control KOA by effective biomechanical methods, cut off this vicious circle, correct the abnormal cooperative contraction mode of knee flexion and extensor muscles, alleviate or relieve patients' clinical symptoms, slow down cartilage damage and delay the development of the disease is one of the research hotspots in recent years. This study focuses on the efficacy and safety of Tai Chi in the treatment of KOA, and how to regulate the treatment of KOA through effective biomechanics, and puts forward the following questions: (1) Whether Tai Chi can reduce the abnormal activation of flexor and extensor muscle group and improve the medial moment of knee joint, improve and delay the disease? (2) Can Tai Chi improve the biomechanical characteristics and gait movement function of lower limbs in patients with KOA?

\section{Method/design}

\section{Study Design $\{8\}$}

This study is a parallel-group, randomized controlled trial conducted by Shuguang Hospital affiliated to Shanghai University of traditional Chinese Medicine in Shanghai, China. A total of 72 patients were recruited and participants were randomly assigned to the control group and the test group at 1:1. This trial scheme has been registered with China Clinical Trials Registry (No. ChiCTR2000040769). Written informed consent will be provided for all participants in this study. The Tai chi group will participant in Tai chi twice a week for eight weeks. The control group will receive health care education. After the 8-week intervention period there will be a 16-week follow-period with no active intervention in either group. The detailed test flow and study design are shown in figures 1 and 2 respectively.

\section{Study Setting and Recruitment}

\section{Study setting $\{9\}$}

The study was conducted at Shuguang Hospital Affiliated to Shanghai University of Traditional Chinese Medicine.

\section{Recruitment \{15\}}

The study would recruit patients through hospital outpatient clinics, the official Wechat account of Shi's Center of Orthopedics and Traumatology (one of China's popular social media platforms) and brochures. Only knee osteoarthritis patients who meet the diagnostic criteria of "guidelines for diagnosis and treatment of Osteoarthritis (2018 Edition)" [23] formulated by the Chinese Academy of traditional Chinese Society in 2018 will be recruited. The recruitment was launched in January 2021.

\section{Participants $\{26 a\}$}


Information and informed consent forms have been prepared in accordance with the guidelines of China registered clinical trial ethics review committee. Potential participants receive both forms at least

1 day before their screening visit. During this visit, a study physician explains all study procedures, and written informed consent is only given after participants had adequate time to ask questions. We include male and female subjects with knee osteoarthritis aged 40-70 in this study. Each subject should voluntarily sign an informed consent form before testing begins in the study.

\section{Eligibility criteria\{10\}}

\section{Inclusion Criteria}

1. In accordance with the diagnostic criteria of medial one-compartment KOA in the "guidelines for the diagnosis and treatment of Osteoarthritis (2018 Edition)" issued by the Joint surgery Group of the Orthopaedic Branch of Chinese Medical Association;

2. The knee joint $X$-ray film was evaluated by 0 grade, $\varangle$ grade, $\nabla$ grade or $\nabla$ grade according to kellgrenlawrence(K-L) grade;

3. Male or female aged 40 to 70 ;

4. Complete walking for at least 10 minutes without assistive devices;

5. Be with a high school and above education background, understanding the requirements of researchers correctly;

6. Agree to and sign the informed consent.

\section{Exclusion Criteria}

1. Do not meet the inclusion criteria;

2. The K-L classification of knee joint was grade IV, or joint deformity, with serious complications affecting knee joint function;

3. Be complicated with serious cardiovascular and cerebrovascular diseases, digestive system diseases, autoimmune diseases, psychosis, acute or chronic infection, or be suffered from other neuromuscular diseases affecting gait;

4. Female with pregnancy and lactation;

5. Patients who had received other treatment in the last 2 months may have a potential impact on the study;

6. Other situations being considered as inappropriate to the participants.

\section{Shedding Criteria: This study can be excluded if one of the following conditions is met.}

1. Those who fail to meet the inclusion criteria and are mistakenly included;

2. Can not accurately and fully understand the research-related problems, resulting in a serious lack of information and data collection; 
3. Subject gives up or asks to quit voluntarily.

\section{Randomization}

\section{Sequence generation $\{16 a\}$}

In this study, a randomized parallel controlled trial design is adopted, and the KOA subjects who meet the test and research criteria are randomly divided into two groups (proportion of 1:1), which are the test group and the control group. The random number table was generated by special statistical staff in Shanghai University of T.C.M.according to strict random procedures by using SAS software (version 9.4, SAS Company, Cary, NC, USA). The randomized list is stored on a secure database (Microsoft Office Access 2007) by the data manager, and is inaccessible by the relevant case observer or other researchers. Participants will only be given one chance to register at random, and no information about KOA subjects will be deleted from the database. This makes the allocation sequence of subjects unpredictable and allows KOA subjects to be assigned to different interventions to prevent selection bias.

\section{Concealment mechanism \{16b\}}

The digital randomization scheme corresponding to serial number 1-72 was generated by the SAS software, which was hidden by a sequentially numbered opaque sealed envelope. After signing the informed consent form, the participants were randomly divided into the test group and the control group according to the order in which they were included in the study.

\section{Allocation and implementation\{16c\}}

Upon the participants signed the informed consent, the diagnostic intervention physician is responsible for asking the person in charge of the random number and corresponding group information, and then the person in charge of clinical immediately asks for the random number and corresponding group code from the "random center" by telephone, and then informs the patient of the random number, corresponding group code, and intervention measures by telephone or WeChat. The intervention physicians are not allowed to disclose the above information to subjects and physicians who collect information about symptoms and signs.

\section{Blinding $\{17 a, 17 b\}$}

Because of the limitations of the treatment methods in this study, participants and researchers were unable to assign treatment blindly. However, in order to reduce the risk of bias in the study, the researchers will apply blind methods to evaluators, data managers and data statistical analysts in result evaluation, data collection and data analysis.

\section{Interventions $\{11 \mathrm{a}\}$}

During the study, subjects prohibited the use of other non-drug or drug treatments for KOA, including acupuncture, needle knife, as well as arthroscopy and surgery. If the subjects need other treatments, they 
need to notify the relevant researchers and obtain approval before using them. Participants should fill out the treatment record card in a timely and objective manner and present it at each agreed follow-up time, along with any symptoms of discomfort and adverse reactions during the treatment, including related drugs they need for other complicated diseases.

\section{Control Group}

Participants in the control group attended a 60-minute group meeting once a week, which would included 30 minutes of health care education, followed by a 30-minute discussion for eight weeks. health care education covers health-related topics, such as diet, daily activities, and fitness tips for osteoarthritis of the knee. Participants in the control group will be asked to maintain their previous lifestyle and will not need to participate in any other regular rehabilitation programs.

\section{Test Group}

Select Tai Chi to starting posture, brush knee and twist step on both side, hold the lute, forearm rollings on both sides, single whip, cloud hands, part the wild horse's mane on both side, closing form. According to the "eight-style Tai Chi standard operating procedures" formulated by the project team members and sports associate professors. According to the tolerance of the subjects, formulate appropriate exercise prescriptions, and guide, teach and supervise the subjects. The subjects concentrated on practicing twice a week and once a day at other times, and filled out the exercise log card for 8 weeks.

\section{Criteria for discontinuing or modifying allocated interventions $\{11 \mathrm{~b}\}$}

Participants have the right to discontinue their participation in the study if they have an adverse reaction, a new change in their condition, or any unexpected situation, whether related to the study or not, during the study period. The researchers will judge the adverse reactions and give additional medical treatment to the participants' diseases. In the study, we will try our best to prevent and treat the injury that may be caused by this study. if the patient withdraws from the study because of adverse reactions or ineffective treatment, participants should take emergency analgesic or sedative, muscle relaxant drugs for intervention. Whether or not to participate in the study is entirely up to you. Participants may refuse to participate in the study, or withdraw from the study at any time during the study, without prejudice to the relationship between the participant and the physician, and without prejudice to the loss of medical or other benefits to you.

\section{Strategies to improve adherence to interventions $\{11 c\}$}

First of all, adequate communication can increase participants' adherence to treatment and help them build confidence in the treatment of the disease. In order to improve the compliance of participants, weekly TCM health lectures will be conducted during the study period to explain the current condition, the long-term nature of treatment and the expected benefits to participants in detail. In addition, the researcher will keep in touch with the participants through telephone or WeChat, so that the participants can effectively understand that every step of treatment in the study is standard and normative, so as to 
improve the participants' self-efficacy in the study. Finally, participants will receive reimbursement for their time and transportation in the form of a gift card.

\section{Relevant concomitant care permitted or prohibited during the trial $\{11 \mathrm{~d}\}$}

In principle, participants are not allowed to use other drugs and treatments for KOA during the study period, including painkillers, muscle relaxants, and acupuncture. Participants taking drugs or treatments for other diseases during the study should be recorded in detail in the CRF. If a participant's condition worsens or does not improve during the study period, you can ask the investigator for alternative treatments and record them in the Treatment Record Form.

\section{Provisions for post-trial care\{30\}}

Once the project is completed, relevant researchers will review the participants' research records if necessary. Participants who have completed clinical trials but are not in remission will be able to continue to receive clinical care or other alternative treatments for the disease from their doctors, such as combined drug therapy, acupuncture and other comprehensive conservative treatment, or arthroscopy, surgery and other treatments. Participants can also be identified as hospitalized patients for further treatment if their knee pain is severe, affecting their sleep, or their quality of life is severely affected.

\section{Outcome Measurements}

\section{Participant timeline $\{13\}$}

The visit time window was $0^{\text {th }}$ day, $4^{\text {th }}$ weekend and $8^{\text {th }}$ weekend during the treatment period, and $12^{\text {th }}$ weekend and $24^{\text {th }}$ weekend of the follow-up after treatment respectively. The basic demographic information data such as age, sex, height and weight of the participants were collected on $0^{\text {th }}$ day and $8^{\text {th }}$ week respectively. The participants' knee joint WOMAC questionnaire, knee static and dynamic palpation information, knee specialized physical examination information and information related to adverse events were collected for each visit. The biomechanical information of the lower limbs of the participants was collected on the $0^{\text {th }}$ day, $8^{\text {th }}$ weekend and $24^{\text {th }}$ weekend respectively.

\section{Outcomes $\{12\}$}

\section{Primary outcome measurements}

\section{WOMAC Score}

The Western Ontario and McMaster University osteoarthritis index (WOMAC), proposed in 1988 for the first time, is a scoring system for hip arthritis and knee arthritis invented by Bellamy and her colleagues. And the reliability, effectiveness and sensitivity of WOMAC scoring scale in evaluating knee joint were objectively evaluated[24]. 
WOMAC score is used to evaluate the severity of arthritis and its therapeutic effect according to the symptoms and signs of patients. From the content point of view, this scale evaluates the structure and function of knee joint from three aspects: pain, stiffness and joint function. There are 24 items in total, including the basic symptoms and signs of osteoarthritis of the whole knee. There are 5 items in pain with 2 items in stiffness and 17 items in joint function. The Visual Analogues Scale(VAS) scale can be used to record the scores, ranging from 0 to 10 . The higher the score is, the worse the limb function is and the higher the score is the more severe the pain is.

\section{Secondary Outcome Measurements}

The Vicon 3D motion capture system (vicon, UK), software version (Nexus1.8.5), 16 ViconT40s infrared high-speed cameras (SamplingSize100Hz) and 4 AMTI 3d force measuring tables (AMTIOR6Series, USA; SamplingSize $1000 \mathrm{~Hz}$ ) and NORAXON(TelemyoDTS, USA)16-channel wireless surface electromyography (Sampling Size1500Hz) were used in the study.

The data used to collect lower limb biomechanics is Cast reverse dynamics model. Static Markers include: bilateral greater trochanter of femur, medial and lateral condyles of femur, medial and lateral ankles, metatarsal heads (1st, 2nd and 5th metatarsal bones), heel, anterior superior iliac spine, posterior superior iliac spine and iliac spine. Dynamic Markers are composed of four cursor points which are not in the same line. Five groups of Cluster cursors are respectively placed at the lateral edges of left and right thighs and legs, and 2 centimeters below the posterior superior iliac spine. Surface electromyography electrodes are placed at the muscle belly for the test. Then, the subject was instructed to practice in the trail for 5 to 10 minutes. After finding a suitable starting position, he/she walked in a uniform natural state and passed through the dynamometer area. The movement track of cursor is collected by 3D motion capture system, and the data of 3D force table and surface electromyography are recorded. When the data is obtained completed, the measurement is repeated at least 10 times.

\section{Muscle Co-contraction}

The surface electromyography (SEMG) data obtained by walking in natural state is converted into the corresponding muscle peak voltage obtained by maximum voluntary contraction (MVIC), so that the percentage of each muscle in MVIC during exercise is obtained. Then, according to the muscle function, they were paired: $\nabla$ external femoral muscle/peroneal muscle, $\nabla$ external femoral muscle/biceps femoris muscle, $\nabla$ external femoral muscle/anterior tibial muscle, $\nabla$ internal femoral muscle/peroneal muscle, and $\mathbb{Q}$ internal femoral muscle/semitendinosus muscle. The formula of co-contraction:

$\mathrm{EMGL} / \mathrm{EMGH} \times(\mathrm{EMGL}+\mathrm{EMGH})$ was used to calculate the co-contraction ratio of knee muscles.(EMGH is a muscle with high degree of activation, and EMGL is a muscle with low degree of activation).

\section{Kinematics Data of the Knee}

Measure the maximum (flexion/extension) range of motion of the sagittal plane of the knee joint when the foot touches the ground, the one at the early stage of the supporting phase and the swing phase 
during the natural walking of the lower limbs; Maximum (varus/valgus) range of motion of the coronal plane of knee joint in support phase and swing phase; Maximum (internal rotation/external rotation) range of motion of knee joint horizontal plane in support phase and swing phase.

\section{Kinetics Data of the Knee}

The peak values (flexion/extension) of the first wave and the second wave in the sagittal plane of knee joint were measured during the natural walking of lower limbs on the AMTI 3D force measuring tables. At the same time, the force moment of coronal plane (varus) of knee joint, force moment of knee joint horizontal plane (internal rotation/external rotation) and knee adduction angular impulse were measured as well.

\section{Follow-up}

To evaluate the short-term efficacy, long-term efficacy and the safety of manipulation therapy, we will follow up the participants on the $8^{\text {th }}$ weekend, as well as the $12^{\text {th }}$ and $24^{\text {th }}$ weekends. The researcher will inform the participants to come to the hospital and collect their basic information, disease-related indicators, biomechanical indicators and safety indicators.

\section{Data collection and management}

\section{Data collection $\{18 \mathrm{a}\}$}

When recruiting the patients, researchers will collect basic demographic information such as age, sex, height, and weight, as well as relevant information about the condition. The knee WOMAC score, static and dynamic palpation information, physical examination information, adverse events and treatment compliance were recorded in each interview. On the $0^{\text {th }}$ day, $8^{\text {th }}$ weekend and $24^{\text {th }}$ week of the visit, the information of lower limb kinematics, dynamics and muscle co-contraction of the participants were collected. Then, two unsuspecting data administrators who did not participate in clinical research will independently receive the completed clinical trial table and input it into an EXCEL database. They need to complete strict training in data monitoring.

\section{Plans to promote participant retention and complete follow-up $\{18 \mathrm{~b}\}$}

To ensure retention of participants, follow-up visits will be scheduled to coincide with routine clinic appointments as far as possible.The study staff will contact participants, either over the phone or WeChat, before their scheduled follow-up appointment, at immediately after the $0^{\text {th }}$ day, and $8^{\text {th }}$ weekend after intervention, and at 12 and 24 weeks after treatment follow-up.

\section{Data management $\{19\}$}

Then they input the data into the China Clinical Trial Registration Center, which will use the electronic data management system to track and monitor the test data of the Science and Technology Department of 
Shuguang Hospital affiliated to Shanghai University of Traditional Chinese Medicine in real time. Finally, the evaluator will complete the human biomechanics test and its data processing and analysis. The data obtained will be compared and analyzed between groups according to the time point of each visit to evaluate and analyze the effectiveness and safety of Tai Chi exercises, to analyze the effects of Tai Chi on the kinematics, dynamics, and muscles of the participants' lower limbs, to analyze the knee joint flexor and extensor muscle group muscle contraction, the characteristics of the stress and strain changes in the medial compartment and their relationship Correlation between changes in condition.

\section{Confidentiality $\{27\}$}

The medical records (CRF, report form, etc.) of the participants will be completely saved in the research center of the project. All the privacy data of the subjects are stored in encrypted protection, only to be seen by the main researchers of the project, only for the research of the project, not for other purposes.

Plans for collection, laboratory evaluation, and storage of biological specimens for genetic or molecular analysis in this trial/future use $\{33,26 \mathrm{~b}\}$

Not applicable in this study.

\section{Statistical Analysis}

\section{Statistical methods $\{20$ a $\}$}

After all the clinical data are collected and summarized, someone will be in charge of inputting the data into Excel according to the serial number of subjects, and inputting the original data into the database by SPSS20.0 statistical software, and carrying out statistical processing and analysis. If it obeys normal distribution or approximate normal distribution, it will be expressed by mean \pm standard deviation (). If it doesn't obey normal distribution, it will be expressed by median $(\mathrm{m})$ and interquartile distance $M((\mathrm{Q}))$. The demographic data and the measurement data of baseline scores between groups were examined by $T$ test for measurement data and the counting data were examined by $X^{2}$ test. WOMAC score, biomechanical data and other observation indicators, the comparison of changes within groups was examined by paired sample t-test, the comparison of differences between groups was examined by two independent sample t-test (corrected t-test). $P<0.05$ indicates that the difference between them is statistically significant.

\section{Methods for additional analyses (e.g., subgroup analyses)\{20b\}}

There will be no other additional analyses beyond the main analyses for the primary and secondary outcomes.

Methods in analysis to handle protocol non-adherence and any statistical methods to handle missing data $\{20 \mathrm{c}\}$ 
All subjects who signed the informed consent form and screened to be qualified for the randomized trial were regarded to be dropped cases once they did not complete the observation period stipulated in the scheme. However, for the subjects who have no more symptoms or stop the treatment on their own during the process will not be regarded as shedding cases even if their treatment time is less than one course of treatment. The following points should be highlighted in the treatment of shedding cases:

1. When the subject withdraws, the researcher should contact the subject, ask for reasons, record the

2. time of the last treatment, and complete the assessment items as much as possible by visiting the subject's home, making an appointment for follow-up, communicate by telephone call and letter, etc.

3. In case of withdrawal from the trial due to adverse reaction and ineffective treatment, the researcher should take corresponding treatment measures according to the actual situation of the subject.

4. For shedding cases, researchers must fill in the detailed reasons for shedding in the case report form.

5. The statistical analysis should be combined with the actual situation. If adverse reactions occur, they should be included in the statistics of adverse reactions.

6. The shedding rate should be controlled less than $10 \%$ as far as possible.

\section{Plans to give access to the full protocol, participant level data,and statistical code $\{31 \mathrm{c}\}$}

The protocol of the study is publicly available on on the website of China Registered Clinical Trial Registration Center with No.ChiCTR2000040769. Due to Chinese laws on privacy protection, the data sets generated and/or analyzed in this study cannot be made public for the time being, but the data will be uploaded to China national knowledge internet (CNKI) within 6 months after the completion of the study and can be viewed on the website. Experimental data can also be obtained by contacting the corresponding author on reasonable request.

\section{Oversight and Monitoring}

\section{Composition of the coordinating center and trial steering committee $\{5 \mathrm{~d}\}$}

This project is directly supervised and managed by the research group of Shanghai University of TCM. The research team and the special case inspector of Shuguang Hospital, the responsible unit of this project, have established a research supervision group, formulated standardized operating standards, and trained the participants in the research, including the communication with patients, the specific implementation process of the treatment plan, the specific implementation of eligibility criteria and shedding criteria, and the statistics and input of observation index, etc. The purpose is to ensure the smooth progress of the clinical research program and the authenticity and objectivity of the clinical data.

\section{Data monitoring $\{21 \mathrm{a}\}$}

The Data and Safety Monitoring Board (DSMB) will be composed of a physician, medical statistician, ethicist, orthopedic surgeon, radiologist and clinical manager and will supervise the study throughout the study period. They will review the clinical treatment methods and all the original data of the project. The 
committee will review the authenticity and reliability of the trial data, as well as the safety of the clinical efficacy. Finally, the committee will report the results of the review and relevant recommendations to the Chief researcher of Shanghai University of TCM.

\section{Interim analyses $\{21 \mathrm{~b}\}$}

In the middle stage of the project, the project research operation SOP, the researcher training manual and the case observation report form will be revised and improved. The interim review meeting of the study will be held, and the data safety monitoring committee reviews the effectiveness and safety of the study intervention, and provides detailed training and explanation to the researchers again. The purpose of the study analysis was to control the bias of the study and ensure the authenticity of the clinical trial results in the middle stage of the project.

\section{Adverse event reporting and harms $\{22\}$}

During the trial, the treatment reactions of all patients were observed and recorded throughout the trial, and the adverse reactions and events occurred during the treatment shall be recorded in detail on the case observation form. Adverse events refer to the following events during the clinical trial: (1) resulting in hospitalization; (2) prolong the length of hospital stay; (3) disability; (4) affect the ability to work; (5) endanger life or death. Synthetically analyze whether the correlation between adverse events and experimental treatment methods is relevant. If an adverse reaction occurs, the clinical observer may decide whether to discontinue the trial based on the patient's situation. Patients who stop treatment due to adverse reactions should be tracked and investigated, and the results should be recorded in detail.

\section{Auditing \{23\}}

This project is directly supervised and managed by the research group of Shanghai University of TCM. The relevant personnel of the research group of Shuguang Hospital and the research quality control personnel of this project have established a research supervision group and formulated the relevant standardized operation standards and rules of the study. Formulate relevant operational standards and standardized actions in the process of clinical research and implementation with specific and clear quality control measures. The training of researchers should be unified so that they are familiar with the implementation plan and master it, as a result, they are able to improve the consistency level of observation and thus improve the reliability of research conclusions. The quality control specialist shall conduct strict quality supervision and control over the whole research process to ensure that the records and reports of research data are consistent with the original data. The research plan, schedule and observation indicators should be detailed and clear. There should be unified regulations on the division of labor, organization and coordination of personnel to ensure the smooth progress of the research.

\section{Sample Size $\{14\}$}

In this study, the test group and the control group are to be set up. According to the improvement of WOMAC score based on previous research, $a=0.05$ (bilateral test), $\beta=0.10$, boundary value 0.08 , sample 
size ratio $k=1$ in both groups. Considering that the sample size loss rate is about $10 \%-20 \%$, the final sample size was 36 .

\section{Plans for communicating important protocol amendments to relevant parties (e.g., trial participants,ethical committees) $\{25\}$}

After careful discussion and modification by members of the research group, the research project was registered on the website of China Clinical Trial Registry, and further improved and modified according to the modification comments provided by the expert group. The approval number was ChiCTR2000040769. The research project has formulated standardized operating standards and procedures, and trained the participants. In order to ensure the smooth progress of the clinical research program and the authenticity and objectivity of the clinical data, each researcher should execute the research plan in strict accordance with the SOP and investigator's manual for the research scheme. This study plan was submitted to the Ethics Review Committee of the Chinese Clinical Trial Registration for review, and was modified and implemented according to experts' opinions. The approval number is ChiECRCT20200335.

\section{Dissemination plans $\{31$ a $\}$}

We plan to disseminate study results through peer reviewed journal publications and conference presentations.Study findings will also be shared with relevant clinical and scientific groups.

\section{Discussion}

With the aging of the social population, the incidence of knee osteoarthritis has risen sharply, and it is currently one of the main diseases that cause chronic knee joint pain, dysfunction, and even loss of labor. A total of 77,957 systematic evaluations of the epidemiology and burden of disease in Chinese KOA from 33 studies showed a high prevalence and burden of disease in KOA [26].

According to traditional Chinese medicine, "Jin" (muscles) and bones (joints) complement each other and influence each other. The imbalance of muscles and bones in the knee is the key core pathogenesis of the occurrence and development of KOA. According to modern muscle and bone biomechanics, the stability and physiological movement of bone and joint are realized jointly on the basis of static balance and relying on the dynamic balance of muscle movement, and the imbalance of dynamic and static system and the deterioration of local and whole mechanical environment of knee joint are the most important reasons to induce and aggravate KOA. Whether it is the theory of "harmony of jin and bone" in traditional Chinese medicine, or the concept of dynamic balance between muscle contractile force (jin) and joint stress (bone) in modern medicine, both of them are fully aware of the importance of muscle and other tissues in the occurrence and development of KOA. Modern clinical biomechanical theory shows that the abnormal cooperative function of muscles in the process of exercise is the main cause of abnormal joint stress. The abnormal co-contraction of the agonist and antagonist muscles driving the motion of the knee joint results in the abnormal increase of the internal moment of the knee joint, while in order to maintain the balance of the torso in the process of movement, the internal and external moment needs to 
achieve dynamic balance. Therefore, the joint produces abnormal motion patterns in the process of movement, and the external moment of the joint is increased to balance the excessive internal moment caused by muscle injury. However, the excessive external moment leads to the abnormal increase of the stress in the medial compartment of the knee joint, which further aggravates the condition and forms a vicious circle.

Studies have shown that effective muscle stretching can reduce the abnormal pattern of muscle cooperative contraction in patients with KOA during exercise, and then reduce the force in the medial compartment of the knee joint, improve the stress condition of the knee joint during exercise, and alleviate clinical symptoms.After 6 weeks of muscle stretching training for KOA patients, Alkhlaifat [27] found that the pain symptoms and walking function of the patients were significantly improved compared with those before treatment, and the co-contraction ratio of the lateral vastus muscle / biceps femoris muscle decreased by $26.68 \%$ and $12.68 \%$ respectively in the early and middle stages of the support phase. And this training includes squat, hip abduction and other postures, which is very similar to Tai Chi movements, such as the starting posture, brush knee and twist step on both side[28]. After muscle stretching training in 21 patients with KOA, Preece et al found that the co-contraction of external femoral muscle/biceps femoris muscle and internal femoral muscle/semitendinosus muscle decreased in varying degrees, and the abnormal mode of joint stress was also improved as compared with that before treatment[29]. Another study [30] showed that relaxing the muscles around the knee joint can not only improve the pain symptoms of patients with KOA, but also effectively improve the strength line of patients'lower limbs, so as to correct the abnormal stress on the joint. Pizzolato[31] observed the force on the knee joint when the human body swings left and right by the real-time feedback system combined with surface electromyography and simulation technique, found that the torso tilts left and right drives the flexion and extensor muscles of the knee joint to co-contraction, corrects the force line of the lower extremities and keeping the force line close to the center of the knee joint, as well as reduces the abnormal stress pattern of the knee joint during movement, which is also consistent with the previous research results of Hunt [32]. In Tai Chi exercises, there are many movements, such as the starting posture, brush knee and twist step on both side, and so on, which include a large number of squatting, hip abduction and other movements to stretch the flexion and extensor muscles of the knee joint, which is very similar to this kind of therapy. Therefore, we infer that the biomechanical mechanism of Tai Chi in the treatment of KOA may be closely related to its ability to improve the co-contraction of knee flexion and extensor muscles after practice. This coincides with the academic viewpoint of KOA's traditional Chinese medicine, which is to "treat bone from jin" from the point of view of "harmony of jin and bone".

To sum up, the scientific hypothesis of this study is that the biomechanical characteristics of patients with KOA medial compartment are abnormal co-contraction mode of knee flexion and extensor muscles, imbalance of external moment and internal moment, and abnormal increase of stress in medial compartment. Tai Chi exercise can adjust the internal moment, improve the mechanical environment around the knee joint, rebuild the balance of muscles and bones, improve or delay the occurrence and development of KOA. In order to test this hypothesis, the following research work will be carried out: (1) To study the efficacy and safety of Tai Chi therapy for KOA. (2) To observe the changes of biomechanical 
parameters, gait parameters and clinical condition of patients before and after Tai Chi treatment of KOA, and to compare and analyze the relationship and influencing factors between them. (3) To study the factors of how Tai Chi therapy performed in KOA, and its biomechanical regulation mechanism of Tai Chi.

\section{Trial Status $\{3\}$}

The treatment protocol version number currently in use is version 1.0, which was revised on 12 December 2020. Recruitment began on 01 January 2021, and the approximatedate for the completion of recruitment will be 31 December 2024.

\section{List Of Abbreviations}

KOA

Knee osteoarthritis;

TCM

Traditional Chinese Medicine

ANOVA

analysis of variance

MRI

magnetic resonance imaging

WOMAC

Western Ontario and McMaster Universities Osteoarthritis Index

VAS

Visual Analogues Scale; SEMG:surface electromyography

MVIC

maximum voluntary contraction

ROM

range of motion

KAM

knee adduction moment

FTA

femorotibial angle

\section{Declarations}

\section{Acknowledgments}

None

\section{Authors' contributions \{31b\}}


All named authors adhere to the authorship guidelines of Trials. All authors have agreed to publication. All authors gave final approval of the version to be submitted. ZH, ZM, PF conceived of the study, and participated in its design and coordination. DG, ZM, CY, YG, LZ, and LJ performed research. PF and ZH wrote and revised the manuscript.

\section{Funding $\{4,5 c\}$}

Shanghai Clinical key specialty "Orthopaedics and Traumatology of traditional Chinese Medicine" (No.shslczdzk03901), National Natural Science Foundation of China(No.81973875), National Natural Science Foundation of China(No.82074466), Pilot Project of Clinical Cooperation of traditional Chinese and Western Medicine in Shanghai \{No.ZXYXZ-201703, ZY (2018-2020)-FWTX-1007\}, Budget Scientific Research Project of Shanghai University of Traditional Chinese Medicine in 2020 (No.2020LK046), Special Project for innovative training of postgraduates of Shanghai University of traditional Chinese Medicine in 2021 (No.Y2021029). Funding body plays no role in the design of the study and collection, analysis, and interpretation of data and in writing the manuscript.

\section{Availability of data and materials $\{29\}$}

The datasets generated and/or analyzed during the current study are not publicly available due to China laws on privacy protection but are available from the corresponding author on reasonable request.

\section{Authors' information}

Pan Fu-wei (1987-), PhD, attending physician, research in clinical osteoarthropathy T.C.M.

\section{Ethics Approval and Consent to Participate $\{24\}$}

The ethics of this trial research scheme was reviewed and approved by China registered clinical trial ethics review committee with approval number ChiECRCT20200335. After careful discussion and modification by the project team, the study plan of this project was registered on the website of the

China Registered Clinical Trial Registration Center and further improved and modified according to the modification opinions provided by the expert team, with approval number ChiCTR2000040769. Each subject should voluntarily sign an informed consent form before testing begins in the study

\section{Consent for Publication \{32\}}

Not applicable.

\section{Competing Interests $\{28\}$}

The authors declare that they have no competing interests.

\section{Author Details $\{5 \mathrm{a}\}$}


1 Shi's Center of Orthopedics and Traumatology, Shuguang Hospital Affiliated to Shanghai University of Traditional Chinese Medicine,Shanghai 201203, China

2 Institute of Traumatology \& Orthopedics, Shanghai Academy of Traditional Chinese Medicine,Shanghai 201203, China

\section{References}

1. Törmälehto S,Mononen ME,Aarnio E,et al.Health-related quality of life in relation to symptomatic and radiographic definitions of knee osteoarthritis: data from Osteoarthritis Initiative (OAI) 4-year followup study. Health and quality of life outcomes. 2018; 16:154.

2. Abramoff B, Caldera FE. Osteoarthritis: Pathology, Diagnosis, and Treatment Options. Med Clin North Am. 2020;104:293-311.

3. Hsu PS, Lin HH, Li CR, Chung WS. Increased risk of stroke in patients with osteoarthritis: a population-based cohort study. Osteoarthritis Cartilage. 2017;25:1026-1031.

4. Caughey GE, Vitry Al, Gilbert AL, Roughead EE. Prevalence of comorbidity of chronic diseases in Australia. BMC Public Health. 2008; 8:221.

5. Calders P, Van Ginckel A. Presence of comorbidities and prognosis of clinical symptoms in knee and/or hip osteoarthritis: A systematic review and meta-analysis. Semin Arthritis Rheum. 2018; 47:805-813.

6. Kluzek S, Sanchez-Santos MT, Leyland KM, et al. Painful knee but not hand osteoarthritis is an independent predictor of mortality over 23 years follow-up of a population-based cohort of middleaged women. Ann Rheum Dis. 2016;75:1749-1756.

7. Veronese N, Stubbs B, Solmi M, et al. Association between lower limb osteoarthritis and incidence of depressive symptoms: data from the osteoarthritis initiative. Age Ageing. 2017;46:470-476.

8. Kye SY, Park K. Suicidal ideation and suicidal attempts among adults with chronic diseases: A crosssectional study. Compr Psychiatry. 2017;73:160-167.

9. Innes KE, Sambamoorthi U. The Association of Perceived Memory Loss with Osteoarthritis and Related Joint Pain in a Large Appalachian Population. Pain Med. 2018;19:1340-1356.

10. Hensor EM, Dube B, Kingsbury SR, Tennant A, Conaghan PG. Toward a clinical definition of early osteoarthritis: onset of patient-reported knee pain begins on stairs. Data from the osteoarthritis initiative. Arthritis Care Res (Hoboken). 2015; 67:40-47.

11. Fu K, Robbins SR, McDougall JJ. Osteoarthritis: the genesis of pain. Rheumatology (Oxford). 2018;57(suppl_4):iv43-iv50.

12. Hsia AW, Emami AJ, Tarke FD, et al. Osteophytes and fracture calluses share developmental milestones and are diminished by unloading. J Orthop Res. 2018;36:699-710.

13. Dimitroulas T, Duarte RV, Behura A, Kitas GD, Raphael JH. Neuropathic pain in osteoarthritis: a review of pathophysiological mechanisms and implications for treatment. Semin Arthritis Rheum. 
2014;44:145-154.

14. Leopold AV, Chernov KG, Shemetov AA, Verkhusha VV. Neurotrophin receptor tyrosine kinases regulated with near-infrared light. Nat Commun. 2019;10:1129.

15. Neogi T, Guermazi A, Roemer F, et al. Association of Joint Inflammation With Pain Sensitization in Knee Osteoarthritis: The Multicenter Osteoarthritis Study. Arthritis Rheumatol. 2016;68:654-661.

16. Arendt-Nielsen L. Pain sensitisation in osteoarthritis. Clin Exp Rheumatol. 2017;35 Suppl 107:68-74.

17. O'Neill TW, Felson DT. Mechanisms of Osteoarthritis (OA) Pain. Curr Osteoporos Rep. 2018;16:611616.

18. Reid KF, Bannuru RR, Wang C, Mori DL, Niles BL. The Effects of Tai Chi Mind-Body Approach on the Mechanisms of Gulf War Illness: an Umbrella Review. Integr Med Res. 2019 Sep;8:167-172.

19. Wang C. Complementary and Alternative Medicine and Osteoarthritis. Int J Integr Med. 2013;1:13.

20. Zeni J A ,Rudolph K ,Higginson J S . Alterations in quadriceps and hamstrings coordination in persons with medial compartment knee osteoarthritis. J ElectromyogrKinesiol, 2010, 20:0-154.

21. Ramsey DK, Briem K, Axe MJ, Snyder-Mackler L. A Mechanical Theory for the Effectiveness of Bracing for Medial Compartment Osteoarthritis of the Knee. Journal of Bone \& Joint Surgery American Volume, 2007, 89:2398-2407.

22. Lewek MD, Ramsey DK, Snyder-Mackler L, Rudolph KS. Knee stabilization in patients with medial compartment knee osteoarthritis. Arthritis Rheum. 2005 Sep;52:2845-53.

23. Chen Weiheng, Liu Xianxiang, Tong Peijian, et al.Consensus of TCM experts in diagnosis and treatment of knee Osteoarthritis (2015 version) [in Chinese].Zhong Yi Zheng Gu,2015,27(07):4-5.

24. Bellamy N, Buchanan WW, Goldsmith CH, Campbell J, Stitt LW. Validation study of WOMAC: a health status instrument for measuring clinically important patient relevant outcomes to antirheumatic drug therapy in patients with osteoarthritis of the hip or knee. J Rheumatol. 1988;15:1833-1840.

25. Lee HY. Comparison of effects among Tai-Chi exercise, aquatic exercise, and a self-help program for patients with knee osteoarthritis. Taehan Kanho Hakhoe Chi. 2006,36:571-80.

26. Wang Bin, Xing Dan, Dong Shengjie, et al.Systematic Review of Epidemiology and Disease burden of knee Osteoarthritis in China [in Chinese].Zhong Guo Xun Zheng Yi Xue Za Zhi,2018, 18(02): 134-142.

27. Al-Khlaifat L, Herrington LC, Hammond A, Tyson SF, Jones RK. The effectiveness of an exercise programme on knee loading, muscle co-contraction, and pain in patients with medial knee osteoarthritis: A pilot study. Knee. 2016 Jan;23:63-9.

28. Tian Wen, Ling Ya, Chi Zhuang, et al.The overall characteristics of tension in Tai Chi [in Chinese]. Zhong Hua Wu Shu(Yan Jiu),2017,6(10):14-18+9.

29. Preece SJ, Jones RK, Brown CA, Cacciatore TW, Jones AK. Reductions in co-contraction following neuromuscular re-education in people with knee osteoarthritis. BMC Musculoskelet Disord. 2016 Aug $27 ; 17: 372$.

30. Jiang Jue, Hu Xiuwu, Tang Runke, et al. Changes of lower limb force lines in patients with knee osteoarthritis treated mainly by manipulation [in Chinese]. Chang Chun Zhong Yi Yao Da Xue Xue 
Bao, 2018,34(01):129-132.

31. Pizzolato C, Reggiani M, Saxby DJ, Ceseracciu E, Modenese L, Lloyd DG. Biofeedback for Gait Retraining Based on Real-Time Estimation of Tibiofemoral Joint Contact Forces. IEEE Trans Neural Syst Rehabil Eng. 2017 Sep;25:1612-1621.

32. Hunt MA, Simic M, Hinman RS, Bennell KL, Wrigley TV. Feasibility of a gait retraining strategy for reducing knee joint loading: increased trunk lean guided by real-time biofeedback. J Biomech. 2011 Mar 15;44:943-7.

\section{Figures}




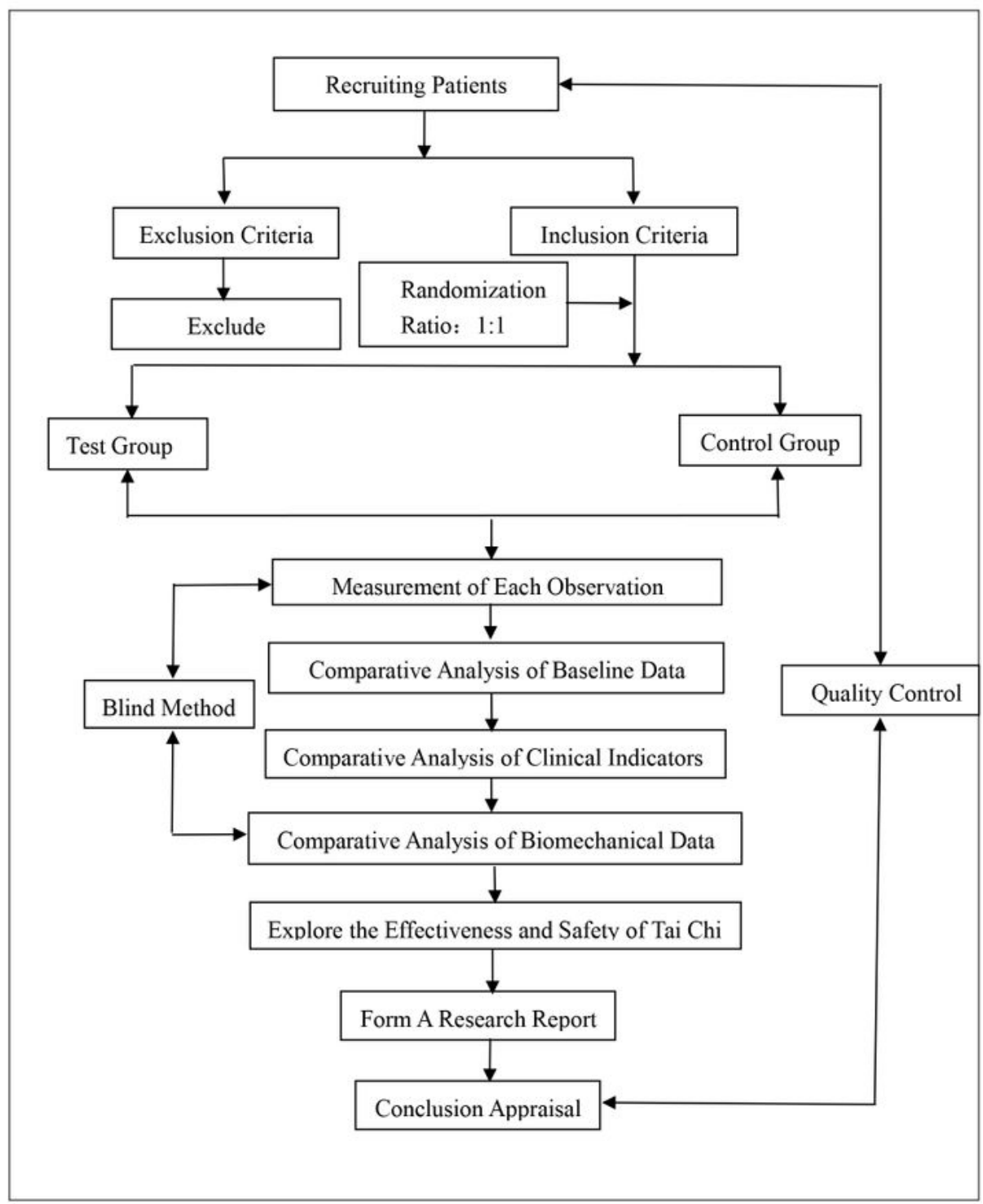

Figure 1

Research flow chart 


\begin{tabular}{|c|c|c|c|c|c|}
\hline Experimental Stage & Screening Period & \multicolumn{4}{|c|}{ Research Period } \\
\hline Follow-up Times & Visit 1 & Visit 2 & Visit 3 & Visit 4 & Visit 5 \\
\hline Time Window (weeks) & $\begin{array}{c}\text { Before } \\
\text { Treatment }\end{array}$ & $\begin{array}{l}\text { The 4th Week } \\
\text { of Treatment }\end{array}$ & $\begin{array}{l}\text { The 8th Week } \\
\text { of Treatment }\end{array}$ & $\begin{array}{l}\text { Follow-up } \\
\text { Week } 12\end{array}$ & $\begin{array}{l}\text { Follow-up } \\
\text { Week } 24\end{array}$ \\
\hline $\begin{array}{c}\text { Collection of Basic Information } \\
\text { and Medical History }\end{array}$ & & & & & \\
\hline Sign the Informed Consent Form & $\bullet$ & & & & \\
\hline Inclusion and Exclusion Criteria & $\bullet$ & & & & \\
\hline $\begin{array}{c}\text { Past Medical History and Treatment } \\
\text { History }\end{array}$ & • & & & & \\
\hline Imaging Examination & - & & & & - \\
\hline Intervention Record & & $\bullet$ & $\bullet$ & & \\
\hline Implementation of Doctor's Orders & & $\bullet$ & $\bullet$ & & \\
\hline \multicolumn{6}{|l|}{ Disease-related Indicators } \\
\hline Demographic Data & - & & & & - \\
\hline Specialized Knee Examination & $\bullet$ & $\bullet$ & $\bullet$ & $\bullet$ & $\bullet$ \\
\hline Dynamic and Static Palpation & - & $\bullet$ & $\bullet$ & $\bullet$ & - \\
\hline WOMAC Questionnaire & $\bullet$ & $\bullet$ & $\bullet$ & $\bullet$ & - \\
\hline $\begin{array}{c}\text { Whole Organ Magnetic Resonance } \\
\text { Imaging Score(WORMS) }\end{array}$ & $\bullet$ & & & & - \\
\hline \multicolumn{6}{|l|}{ Biomechanical Indicators } \\
\hline $\begin{array}{c}\text { Co-contraction Ratio of Flexion and } \\
\text { Extension Muscles }\end{array}$ & - & & $\bullet$ & & - \\
\hline Kinetics Data of the Knee & $\bullet$ & & $\bullet$ & & $\bullet$ \\
\hline Kinematics Data of the Knee & $\bullet$ & & - & & - \\
\hline \multicolumn{6}{|l|}{ Safety Observation } \\
\hline Adverse Events/Reactions & & $\bullet$ & $\bullet$ & $\bullet$ & $\bullet$ \\
\hline \multicolumn{6}{|l|}{ Other Work } \\
\hline Compliance/Safety Evaluation & & $\bullet$ & $\bullet$ & $\bullet$ & $\bullet$ \\
\hline $\begin{array}{l}\text { Record Whether There is A } \\
\text { Combination of Treatment }\end{array}$ & • & $\bullet$ & $\bullet$ & - & $\bullet$ \\
\hline Research Summary & & & $\bullet$ & & $\bullet$ \\
\hline
\end{tabular}

\section{Figure 2}

Recommended content for the schedule of enrolment, interventions, and assessments

\section{Supplementary Files}

This is a list of supplementary files associated with this preprint. Click to download. 
- SPIRITChecklist.pdf

Page 24/24 